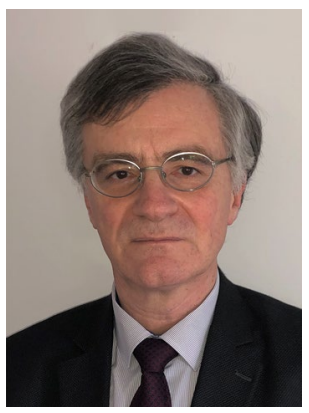

\title{
COVID-19 research and science in the service of public health: the example of Greece
}

\author{
The COVID-19 response in Greece has been swift and flexible, adapting to the evolving \\ pandemic and creating unique opportunities for research.
}

$\Lambda$ midst a third surge in SARS-CoV-2 infections, Greece is confronting again the heavy burden of COVID-19 on people, healthcare workers, the health system, and society as a whole. We have experienced, along with the rest of the world, what this virus is capable of doing, how fast it can spread in vulnerable populations, and how it can "chew up" the intensive care unit (ICU) capacity at the local level. Physicians from multiple subspecialties wore the infectious disease hat and entered the "battlefield". Flexibility and sustainability of care remain essential parts of the response.

As scientists, we did not shy away from the opportunity to conduct research to understand and develop ways to fight the new virus causing this pandemic. From basic science and biology to epidemiological and clinical (namely, to the bedside) applied research, we have all seen the fruits of this tireless effort, especially in the availability of efficient vaccines.

As in the rest of the world, scientists in Greece grabbed the opportunity to engage in epidemiological, basic and clinical research. Epidemiology (using data from the National Public Health Organization of Greece, that is, the Greek CDC) and clinical science were at the forefront, from reports on travel-related outbreaks to serological surveys verifying the spread of an under-reported and underdiagnosed disease. Mathematical modeling estimated the effects of non-pharmaceutical interventions, and artificial intelligence algorithms were implemented to screen travelers in a cost-effective manner when there was limited testing capacity. Our clinical centers, which were heavily involved in clinical trials evaluating biomarkers and the therapeutic use of new and repurposed medications, faced the key challenge of providing care while supporting clinical trial procedures. Epidemiology and molecular science worked in harmony, first in studying the origin of the virus and then in assessing how the virus was introduced to the country through the use of phylogenetic tools. Sewage surveillance was used as a new epidemiological tool to assess viral circulation and load in the community and to screen for new virus variants.

A central paradigm of immunity is that interferon (IFN)-mediated antiviral responses precede proinflammatory ones, optimizing host protection and minimizing collateral damage. Using existing databases, data repositories and biobanks (not only for COVID-19 but for other respiratory pathogens, like influenza), we proposed a potentially unique therapeutic role for interferon- $\lambda$ and revealed an environment of an "untuned" antiviral response due to the new virus, contributing to persistent viral presence, hyperinflammation and respiratory failure, which are not as common with the usual seasonal influenza enemy.

Understanding the mechanisms mediating severe disease is at the core of developing new therapies. A national research program was established soon after the start of the pandemic in Greece encompassing a host-genetics study that is part of a global consortium (in collaboration with the International Common Disease Alliance scientific forum, details available at https://www.covid19hg.org/partners/). Developing anti-SARS-CoV-2 monoclonal antibodies to facilitate expanded testing and therapy and conducting viral surveillance and virome analysis to detect virus variants and escape mutants while rolling out the vaccine are parts of this national project. In the epidemic setting, some of this work will continue to inform contact tracing and other public health policies and actions over the next year. The pandemic has expanded the horizon for scientific research questions in the same way HIV led to a new era in virology. Several questions remain unanswered, especially in relation to immune responses and transmission in the context of immunization.

As in other countries, the pandemic posed considerable problems in the funding and completion of ongoing research. Recruitment of patients to clinical studies and clinical trials was slowed, while travel and customs restrictions limited the timely delivery of reagents and the ability of researchers to equip their laboratories. Moreover, there were delays in the announcements of new grants, due to, among other reasons, the limited availability of personnel working on-site because of parental and health-related leave. On the other hand, funding was increased for research proposals dedicated to COVID-19, but this budget was limited. This mobilized the research community to engage in research related to the pandemic.

At the societal level, the new pandemic has already added its own "dialect" to our everyday life in Greece. Terms like mRNA, neutralizing antibodies, memory T cells, surveillance, $R_{\mathrm{t}}$, rapid antigen testing, variants of concern, vaccine rollout, non-pharmaceutical measures and lockdown have become terms of daily reference not only for scientists but for the general public. Unfortunately, all of this polyphony has unwittingly and unintentionally, for the most part, degraded in several instances into a cacophony of mixed messages and, even worse, into fake news, jeopardizing the efforts to combat the pandemic. The perception of risk created huge psychological issues, especially for people who were socially isolated, leading to increased rates of anxiety and depression. Fear and panic have been and will always be the worst advisors. Communication of research findings to the general public and reliable indicators of its effectiveness are lagging behind basic science research. All of this miscommunication may hurt science and the public's perception of it. At the same time, the pandemic has stimulated the interest of the public in the sciences, the national health care system and public health actions. In this environment, there is a chance for the scientific community to speak up more and to increase the knowledge of the public in basic scientific concepts as they relate not only to infectious diseases but to other enemies of our wellbeing.

In its own way, the pandemic will hopefully be an opportunity for large-scale 


\section{WORLD VIEW I SERIES}

interventions to improve public health in general, not only in Greece but in the entire world. Basic science remains a key player by providing solutions that sometimes appear to come almost in real time. I hope that the appreciation and real-life benefits of all of the amazing scientific work that we have witnessed during the last year will influence investment in research infrastructure in the immediate future, both in our country and elsewhere. This will lead to new discoveries and knowledge, which are expected to bring significant long-term beneficial effects for our health.

Sotirios Tsiodras (iD)

4th Department of Internal Medicine, National and
Kapodistrian University of Athens Medical School, Athens, Greece.

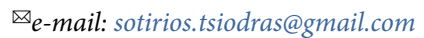

Published online: 13 April 2021

https://doi.org/10.1038/s41590-021-00919-z

Competing interests

The author declares no competing interests. 